\title{
Cyto- and myeloarchitectural brain atlas of the pale spear-nosed bat (Phyllostomus discolor) in CT Aided Stereotaxic Coordinates
}

\author{
Susanne Radtke-Schuller ${ }^{1,2}$ (]) $\cdot$ Thomas Fenzl $^{4} \cdot$ Herbert Peremans $^{5} \cdot$ Gerd Schuller $^{3} \cdot$ Uwe Firzlaff $^{1}$
}

Received: 13 April 2020 / Accepted: 29 August 2020 / Published online: 16 September 2020

(C) The Author(s) 2020

\begin{abstract}
The pale spear-nosed bat Phyllostomus discolor, a microchiropteran bat, is well established as an animal model for research on the auditory system, echolocation and social communication of species-specific vocalizations. We have created a brain atlas of Phyllostomus discolor that provides high-quality histological material for identification of brain structures in reliable stereotaxic coordinates to strengthen neurobiological studies of this key species. The new atlas combines high-resolution images of frontal sections alternately stained for cell bodies (Nissl) and myelinated fibers (Gallyas) at 49 rostrocaudal levels, at intervals of $350 \mu \mathrm{m}$. To facilitate comparisons with other species, brain structures were named according to the widely accepted Paxinos nomenclature and previous neuroanatomical studies of other bat species. Outlines of auditory cortical fields, as defined in earlier studies, were mapped onto atlas sections and onto the brain surface, together with the architectonic subdivisions of the neocortex. X-ray computerized tomography (CT) of the bat's head was used to establish the relationship between coordinates of brain structures and the skull. We used profile lines and the occipital crest as skull landmarks to line up skull and brain in standard atlas coordinates. An easily reproducible protocol allows sectioning of experimental brains in the standard frontal plane of the atlas. An electronic version of the atlas plates and supplementary material is available from https://doi.org/10.12751/g-node.8bbcxy
\end{abstract}

Keywords Chiroptera $\cdot$ Phyllostomatidae $\cdot$ Cytoarchitecture $\cdot$ Myeloarchitecture $\cdot$ AChE $\cdot$ NADPH

\section{Introduction}

The pale spear-nosed bat Phyllostomus discolor (Wagner 1843 ) is a medium sized microchiropteran bat with a geographic distribution ranging from Central America to the northern part of South America (Kwiecinski 2006). Body size of adult animals is $\sim 10 \mathrm{~cm}$ with a wing span of $\sim 42 \mathrm{~cm}$.

Susanne Radtke-Schuller

gsfjj.schuller@online.de

1 Lehrstuhl für Zoologie, Technical University Munich, Freising, Germany

2 Department of Psychiatry, University of North Carolina At Chapel Hill, Chapel Hill, NC 27599, USA

3 Division of Neurobiology, Department Biology

II, Ludwig-Maximilians-University Munich, Planegg-Martinsried, Germany

4 Klinikum für Anästhesiologie und Intensivmedizin am Klinikum Rechts der Isar, TU München, Munich, Germany

5 Department of Engineering Management, University of Antwerp, Antwerp, Belgium
Based on volumetric comparisons of brain structures in bats, Phyllostomus discolor with its relatively large neocortex and high encephalization index has been classified as belonging to the group of 'progressive' chiroptera as opposed to 'basal chiroptera' (Pirlot and Stephan 1970; Stephan and Pirlot 1970). Phyllostomus discolor uses echolocation for orientation and hunting, but also olfaction and vision when lighting conditions are appropriate. Phyllostomus discolor is omnivorous, but mostly feeds on fruit, nectar and pollen and occasionally on insects. Echolocation calls are short ( $1-3 \mathrm{~ms})$, downward frequency modulated $(90-40 \mathrm{kHz})$, multiharmonic and are emitted through the nostrils. Phyllostomus discolor roosts predominantly in hollow trees in colonies up to 400 individuals of both sexes (Kwiecinski 2006) (Fig. 1).

Phyllostomus discolor is well established as an animal model for behavioral and neurobiological research on echolocation and social communication. The peripheral and central auditory system, the vocal motor system and the visual system of Phyllostomus discolor have been the subject of intense research over the last decades (peripheral and 


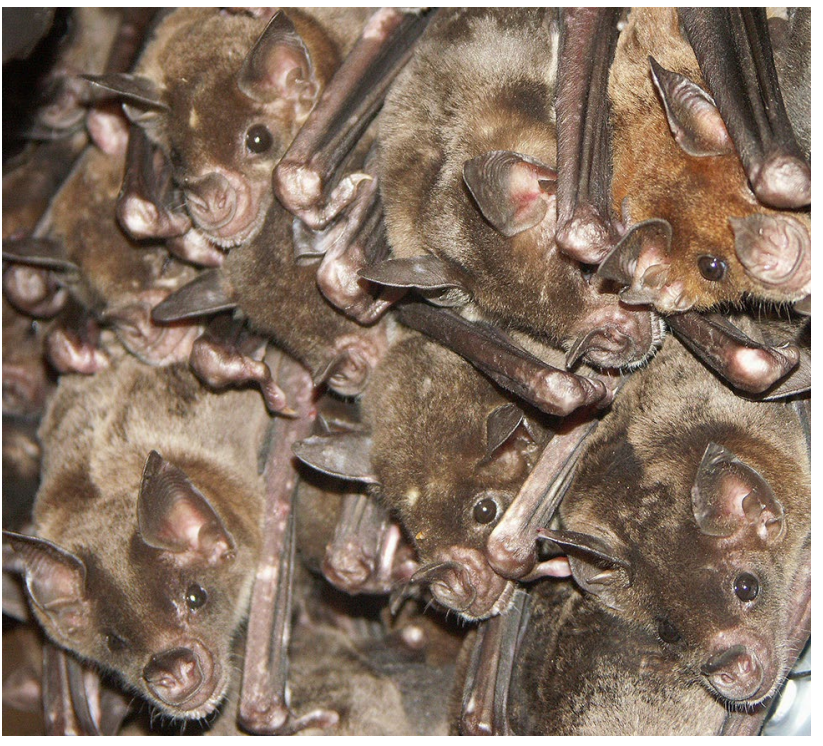

Fig. 1 Phyllostomus discolor in the roost

central auditory system: Bartenstein et al. 2014; Esser and Kiefer 1996; Firzlaff et al. 2007; Firzlaff and Schuller 2003; Goerlitz et al. 2008; Greiter and Firzlaff 2017a, b; Heinrich et al. 2011; Hoffmann et al. 2008a, b; Linnenschmidt and Wiegrebe 2019; Vanderelst et al. 2010; vocal motor system: Fenzl and Schuller 2002; Fenzl and Schuller 2005; visual system: Hoffmann et al. 2016, 2019; Kugler et al. 2019; Rother and Schmidt 1982). However, as no brain atlas for Phyllostomus discolor has previously been published, only one of the earlier studies mentioned above involved neuroanatomy beyond basic localization of recording sites. The single exception was a study of the auditory cortex (AC) in Phyllostomus discolor (Hoffmann et al. 2008b) that identified several AC subfields based on both neurophysiological and neuroanatomical criteria.

A brain atlas is also essential for comparative studies. Phyllostomus discolor has become a valuable animal model in studies of the evolution of species-specific communication (Esser 1994; Esser and Schmidt 1989; Lattenkamp et al. 2018). Phyllostomus discolor is one of the bat species recently discovered to be capable of vocal learning (Knörnschild 2014; Vernes and Wilkinson 2020), a trait observed in only few mammalian species (Janik and Slater 1997; Tyack 2020). New approaches, including the use of molecular genetic techniques, aim to identify the neuronal substrates and brain structures underlying this behavior, for example by mapping the distribution of language related genes FoxPl, FoxP2 and CntnaP2 (Rodenas-Cuadrado et al. 2015, 2018).

The stereotaxic brain atlas of this species will enable scientists from multiple disciplines to reliably target brain structures in neurophysiological and connectional studies, and to align and compare their data with the results of immunohistochemical and molecular genetic studies utilizing a standardized anatomical representation of the Phyllostomus discolor brain.

Bats have a highly diverse biology and anatomy which is also observed in their brain structures, and the macromorphological differences are such, that it is nearly impossible to describe a 'typical bat brain' (Schneider 1957). This underlines the necessity for a distinct brain atlas of Phyllostomus discolor.

However, although there is considerable variety in chiropteran brains, in the preparation of our stereotaxic brain atlas for Phyllostomus discolor, we referred to previous brain atlases of several other bat species that were used for comparison. A cytoarchitectural atlas of the common vampire bat, Desmodus rotundus murinus, a species closely related to Phyllostomus discolor, presents frontal sections with a combined nerve fiber and cell stain (lugol fast blue-cresyl violet) and many delineated brain structures. However, although somewhat useful, there are some limitations of this earlier atlas, including poor representations of brain areas in the histological material and the absence of stereotaxic coordinates (Bhatnagar 2008). An atlas of the short-tailed fruit bat, Carollia perspicillata (Scalia et al. 2013), a species also closely related to Phyllostomus discolor, uses NeuN and Nissl as cell stains for quality sections and depicts comprehensible structural delineations of anatomical structures. High resolution frames for thalamus and amygdala are also added to this atlas. However, unfortunately, this atlas is restricted to the forebrain and also lacks a stereotaxic reference frame. A third atlas we consulted is the three-dimensional digital brain atlas of the mustached bat, Pteronotus $p$. parnellii (Washington et al. 2018) which is an MRI atlas with labels for gross brain structures. Valuable information was also gathered from the atlas of the microchiroptera brain of Myotis montivagus, a 'basal chiroptera', and the megachiropteran Rousettus amplexicaudatus from Baron et al. (1996) and Rousettus aegyptiacus (Schneider 1966).

The stereotaxic brain atlas of the bat Phyllostomus discolor presented here combines high-quality histological material for identification and delineation of brain structures with X-ray computerized tomography (CT). The latter yields internal contours and outlines of the skull which helped to calibrate the relationship between brain and skull coordinates. The brain atlas provides a common reference frame in stereotaxic coordinates for data from different experiments and laboratories, making it possible to reliably target brain structures for a wide range of experimental approaches. A clearly written and easily reproducible protocol provides instructions and procedures for sectioning experimental brains in the frontal plane of the atlas. 


\section{Methods and results}

\section{Animals}

Five adult spear-nosed bats (Phyllostomus discolor, body weight: $40-49 \mathrm{~g}$ ) were used for this study. The animals originated from a breeding colony in the Department of Biology II of the Ludwig-Maximilians-University in Munich. The brain of one animal was processed for cyto- and myeloarchitectural features for the atlas plates. The brains of three more animals were processed for the detection of the calcium-binding proteins parvalbumin, calbindin and calretinin, and acetylcholineesterase, acetylcholine-transferase, NADPH-diaphorase and zinc to gather additional information for the labeling of brain structures. Two additional brain series from neurophysiological experiments stained for cytochrome oxidase were available for comparison. A CT scan of the head of one animal was performed to establish the relationship between the stereotaxic coordinates of brain structures and skull coordinates.

All experiments were conducted in accord with the NIH "Guide for the Care and Use of Laboratory Animals" (2011) and also performed in agreement with the principles of laboratory animal care and the regulations of the "German Law on Animal Protection" (209.1/211-2531-68/03 Reg. Oberbayern).

\section{CT imaging}

The head of the animal was fixed and preserved in $4 \%$ paraformaldehyde (PFA) and then dried for approximately 20 min before scanning with a Skyscan 1076 microCT machine (Bruker, Kontich, Belgium) at the MCT group of the University Antwerpen (Belgium). Scans were performed with a resolution of $35 \mu \mathrm{m}$. The resulting shadow-images were processed with conebeam reconstruction software that accompanies the scanner, using a Feldkamp Reconstruction algorithm.

\section{Histology}

Three animals were perfusion-fixed with $4 \%$ PFA. For the detection of zinc, another animal was perfused with a solution of the modified Timm method according to Danscher (1981). All animals were deeply anaesthetized with Barbital (16 $\mathrm{mg} / \mathrm{mL}$ solution, $0.1 \mathrm{~mL} / 10 \mathrm{~g}$ body weight). When a deep anesthetic state was reached, marked by a complete loss of the flexor reflex at lower limbs and wings, the animals were perfused transcardially with $0.9 \%$ saline (supplemented with $0.1 \%$ heparin) followed by $4 \%$ PFA (in $0.05 \mathrm{M}$ PBS, $\mathrm{pH}$ 7.4).

The angle between the head and body axis during perfusion was carefully positioned in the atlas animal, to be about $110^{\circ}$ since this angle influences the macroscopic orientation of the most caudal part of brainstem and spinal cord (and consequently influences the sectioning plane of the brain most caudally). The brain used for the atlas was postfixed in the skull with $4 \%$ PFA (in $0.05 \mathrm{M}$ PBS, pH 7.4) at $4{ }^{\circ} \mathrm{C}$ for 7 days, to best preserve the brain shape before removal and processing for cyto- and myelinated fiber architecture. The other brains were postfixed up to $24 \mathrm{~h}$. Cryoprotection for freeze cutting was achieved by soaking the brains in $30 \%$ sucrose in $0.05 \mathrm{M}$ phosphate buffer solution for $12 \mathrm{~h}$. The brains were cut on a cryostat (LEICA CM 3050S, Leica Biosystems, Wetzlar, Germany) into four series of $40 \mu \mathrm{m}$ thick frontal sections. The stain for acetylcholine-esterase was performed according to Hedreen (Hedreen et al. 1985). The NADPH-diaphorase stain followed the protocol of Vincent and Kimura (Vincent and Kimura 1992). The sections of the atlas brain were directly mounted on gelatin-coated slides and dried overnight. Alternating section series were stained on-slide either for cell bodies (Nissl) or for myelinated fibers (Gallyas 1979). Sections were imaged with a virtual slide microscope (VS120 S1, Olympus BX61VST, Olympus-Deutschland, Hamburg, Germany) at $10 \times$ magnification using the proprietary software dotSlide ${ }^{\circledR}$ (Olympus).

\section{Atlas coordinate system}

The coordinate system of the Phyllostomus discolor brain atlas follows the conventional definition of anatomical sectioning planes in which frontal sections ('sp' in Fig. 2) are cut perpendicular to the brainstem axis (Fig. 2). In Phyllostomus discolor, the brainstem axis parallels the horizontal tangential plane passing through the most dorsal points of the cerebrum and the most dorsal point of the cerebellum (Fig. 2). This plane is chosen as the origin for the dorsoventral dimension of the coordinate system, with negative values in the ventral direction. The medio-lateral dimension is zeroed to the midsagittal plane (Fig. 2) with negative values on the right side, and positive values towards the left side of the head. There are two sets of coordinates for anterior-posterior position: the anterior-posterior position of the atlas brain plates is indicated relative to the rostral beginning of the neocortex $\left(y_{\text {Start of Neocortex }}=0\right.$; increasing values from anterior to posterior levels) and additionally, coordinates are given relative to the occipital crest $\left(y_{\text {Occipital crest }}=0\right.$; with decreasing values from caudal to rostral levels) as an external skull landmark (Fig. 3).

\section{Stereotaxic reference system}

The coordinate system of the brain atlas was chosen to provide a viable baseline orientation of brain and head in a stereotaxic device. This orientation of the head enables a 


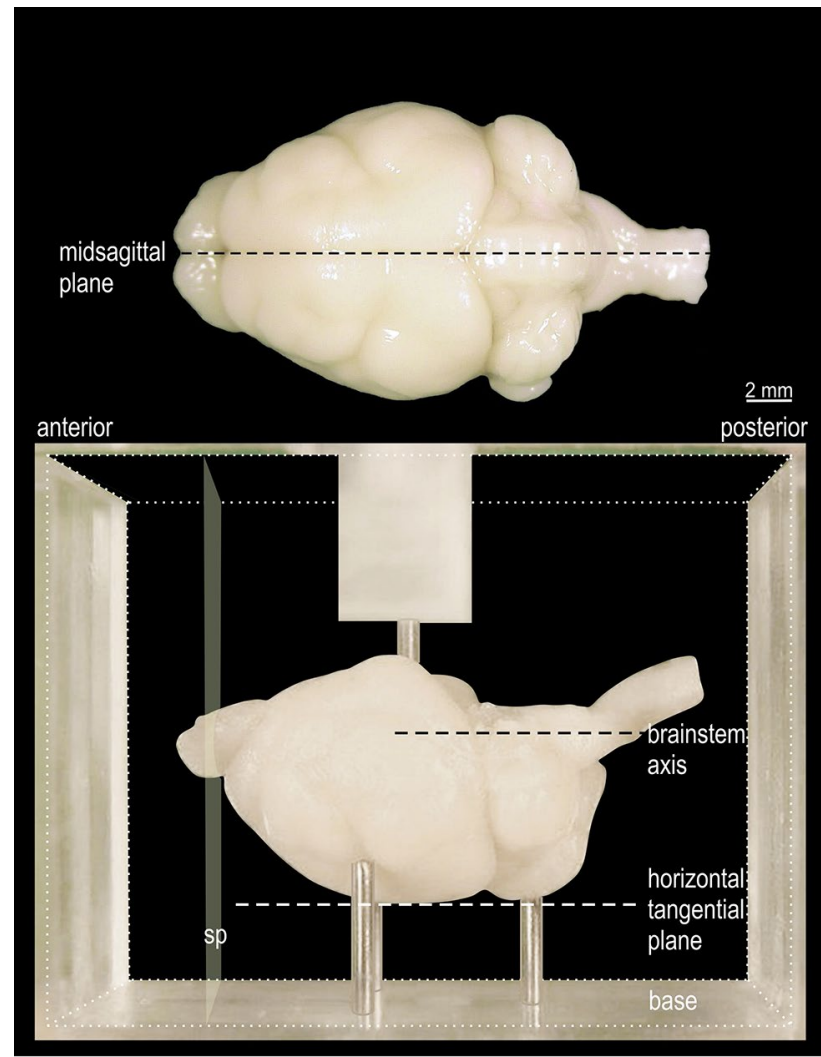

Fig. 2 View of fixed Phyllostomus discolor brain, positioned for embedding. In the lower part of the figure the brain is shown in the acrylic glass box used for embedding (cubical volume indicated by fine dotted lines, front and back walls removed). The brain is positioned on 3 pins protruding from the base so that the plane defined by the most dorsal elevation of cerebrum and cerebellum (horizontal tangential plane) as well as the axis through the brainstem, are aligned parallel to the base. The anterior and posterior surfaces of the embedding block define the frontal sectioning plane (sp) perpendicular to the horizontal tangential plane and to the brainstem axis. A pin protruding from a bracket over the front and back walls of the box (only partly shown) prevents brain movement when the embedding medium is poured into the box. In the upper part of the figure the mid-sagittal plane is drawn in a top view of the brain

comfortable positioning of the animal and is suitable for electrophysiological experiments, tracer injections and optogenetic approaches. The alignment of the skull in the atlas brain coordinate system has been defined via CT. For this purpose, the CT scan was re-sliced in standard coordinates into $50 \mu \mathrm{m}$ thick frontal slices with the open source program AMIDE: A Medical Imaging Data Examiner (amide.exe 1.0.4, (CAndreas Loening, https://amide.sourc eforge.net/; GNU GPL).

Characteristic parasagittal outer skull profile lines of the $\mathrm{CT}$ are accessible during experiments and can be used to

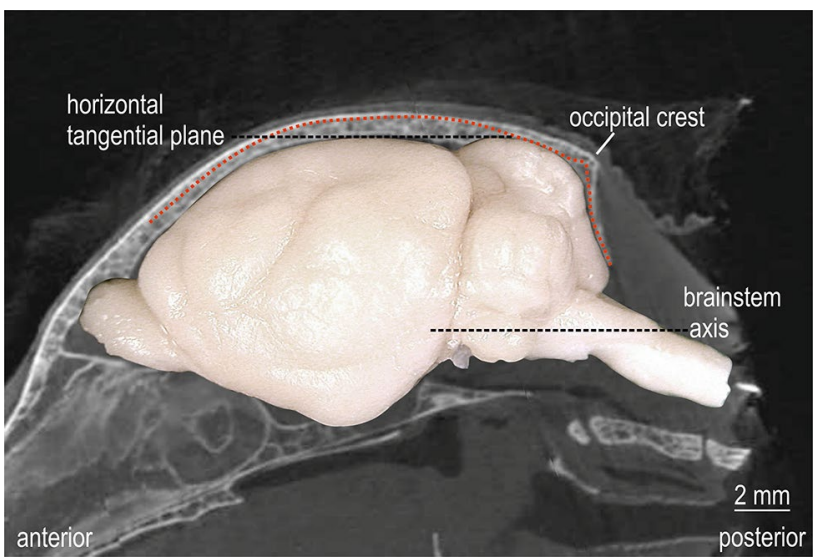

Fig. 3 Atlas coordinate system and stereotaxic reference system. Montage of CT image of mid-parasagittal skull with the abrupt decline of occipital crest and the side-view image of the brain in standard orientation of the atlas. The red dotted line corresponds to the outer skull profile of the parasagittal CT image $1000 \mu \mathrm{m}$ lateral to midline. The horizontal tangential plane through the most dorsal points of cerebrum and cerebellum and the brain stem axis is indicated by black dashed lines

determine the baseline orientation of the skull. The occipital crest marks the abrupt decline of the occiput and is used as stereotaxic origin for the anterior-posterior coordinate (occipital crest, $y_{\text {Occipital crest }}=0$ ). The parasagittal profile line and occipital crest coordinate, together with the symmetrical medio-lateral profile of the skull, define the baseline orientation of the skull in vivo to achieve a best fit of the brain in atlas coordinates. This profile-oriented stereotaxic procedure was described in detail in Schuller et al. (1986) and is recommended as standard adjustment procedure. It has been successfully used in Phyllostomus discolor in many studies involving forebrain and midbrain structures (Borina et al. 2008, 2011; Bartenstein et al. 2014; Fenzl and Schuller 2002, 2005; Firzlaff and Schuller 2007; Firzlaff et al. 2006, 2007; Greiter and Firzlaff 2017a, b; Genzel et al. 2015; Heinrich et al. 2011; Hörpel and Firzlaff 2019; Hoffmann et al. 2008a, b, 2010, 2013, 2015, 2016, 2019). These studies used a lab-internal series of Nissl stained sections that were relocatable to the present atlas series and fitted to the skull profile used here. The skull profile also matches well the contours of the cranium of Phyllostomus discolor presented by Kwiecinski (2006).

The stereotaxic standard position can be maintained throughout a series of experiments from the very beginning, by initially scanning the skull profile, then performing the experimental procedures in the stereotaxically oriented brain, and finally sectioning the brain in the same stereotaxic orientation as provided in the brain atlas. For this purpose 
the fixed brains are embedded in an acrylic glass box (Fig. 2, lower part) following a well-defined protocol (see RadtkeSchuller et al. 2016).

The protocol for positioning the brain for sectioning is straightforward. In short, the brain is positioned on the supporting needles of the embedding case so that the midsagittal plane of the brain is aligned parallel to the long side of the chamber, and that the virtual plane comprising the most dorsal points of the cerebrum and the cerebellum is parallel to the bottom plane of the embedding chamber. The latter adjustment provides a reproducible frontal sectioning orientation, whereas the former is important for the left-right symmetry of the sections. After stabilization of the brain in the desired position by a holding needle in a bracket from the top of the chamber, the embedding medium (a freshly prepared gelatin-albumin-glutaraldehyde or egg-yolk-glutaraldehyde mixture) is poured into the volume around the brain. Hardening of the block takes about 2-3 min. As the side walls, the holding bracket and the adjustable needles of the embedding chamber are detachable, the block can easily be removed from the box. After shock freezing in dry ice the block is directly mounted with its hind surface on the cutting platform of the cryostat. Due to the prior orientation of the brain in the embedding chamber the desired frontal sectioning plane is reached without further adjustment of the block in the cryostat. Further details of the embedding chamber and the procedure (developed for another bat species) can be found in Schuller et al. (1986).

Alternatively, it is also possible to section the brain in the standard atlas plane without embedding. In this case the brain is positioned upside down on a flat surface so that it is seated with the cerebellum and cerebrum on the base. Then part of the brain is cut off perpendicular to the base to create a surface for mounting the brain's portion of interest on the cryostat platform. By subsequent sectioning of the brain parallel to this cutting surface the resulting sections correspond best to the frontal plane of the atlas.

\section{Selection of atlas series and preparation of plates}

The series for the new atlas consists of high-quality histological sections stained for cells (Nissl) and myelin (Gallyas) and matches the previous, successfully used, unpublished series.

To assess how representative the atlas series is within the pool of available Phyllostomus dicolor brain series $(N=7)$, the distance between rostral start and caudal end of the cortical hemispheres was evaluated for comparison. The average of this distance amounted to $11.093 \mathrm{~mm}$ with a standard deviation of $0.544 \mathrm{~mm}$. The distance in the atlas brain is
$11.086 \mathrm{~mm}$, which signifies that the atlas brain is a valid representative of the average sized brain in this bat species. The comparison of the atlas brain series with the CT indicated a shrinkage of $8-9 \%$, so that the virtual in vivo thickness of the sections $(40 \mu \mathrm{m})$ amounts to $43.75 \mu \mathrm{m}$. This shrinkage is in the generally observed range for cryo-protected frozen-cut brains with PFA fixation.

There are 49 plates in the atlas. Nissl-stained and adjacent myelin-stained sections (Gallyas) at equidistant intervals of $350 \mu \mathrm{m}$ (every 8th section) and every 7th CT section were taken to represent the 49 anterior-posterior (ap) levels. Distortions of the sections due to histological processing were compensated by cautiously adjusting the sections to optimize the congruency between outlines of histological sections and appropriate CT slices. Slight differences between the adjusted histology sections and the corresponding CT images do remain and can be judged by the overlay of structural delineations onto the $\mathrm{CT}$ as represented on the abbreviation subplates of the atlas. In the most caudal atlas plates (plate \# > 45) the sections were less commensurate to the CT slices as the head/body angle of the atlas animal during perfusion fixation slightly deviated in orientation from that of the CT scan.

The contrast and brightness of the images of the sections were corrected with Photoshop (CS6, Adobe Systems, San Jose, CA, USA). The images were arranged in the atlas coordinate frame using CorelDraw graphics suite version 20 (2018) (Corel Corporation, Ottawa, ON, Canada). All outlines and delineations were drawn in CorelDraw on the base of the Nissl-stained section of each atlas plate. The structural boundaries seen in the corresponding myelin-stained section generally correlate well with these outlines.

An overview of the plate location in side and top view of the brain is presented in Fig. 4.

The plate at each ap-level consists of four subplates, as illustrated in the examples of Fig. 5a-d. The first subpanel depicts a montage of the Nissl stained half-section with the mirrored adjacent myelin-stained half-section (Fig. 5a). The second subpanel combines the Nissl stained half sections with delineations of the anatomical structures on the mirrored translucent (30\%) Nissl section (Fig. 5b). The third subplate consists of an abbreviation list and the CT slice with the contours of the anatomical structures (Fig. 5c). The forth subplate shows the myelin-stained half sections with Nissl-derived delineations of the anatomical structures superimposed onto the mirrored translucent (30\%) myelinstained half section Fig. 5d).

The inset in the upper left corner of subpanels indicates the anterior-posterior plate location in a lateral brain view which is also shown in numerical form relative to the most rostral "Start of Neocortex", as well as relative to the 


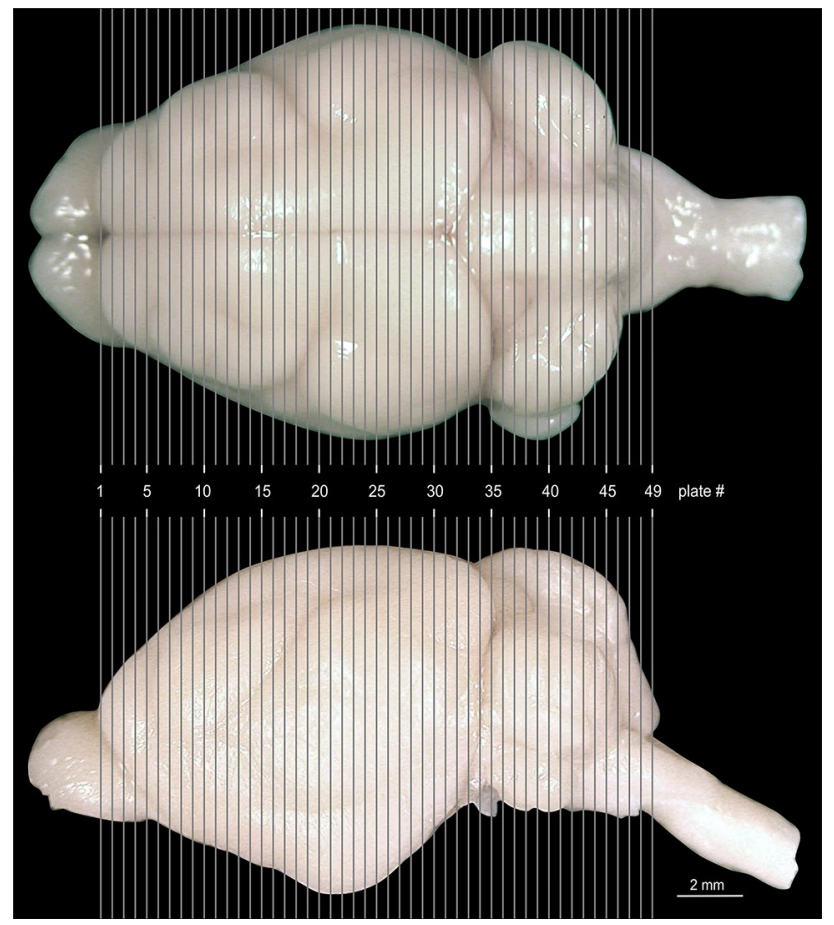

Fig. 4 Anterior posterior location of the atlas plates on the Phyllostomus discolor brain indicated by gray gridlines. Top: view from above, bottom: side view. Distance between plates is $350 \mu \mathrm{m}$

"Occipital crest" in the lower right corner of the 3 subplates $5 \mathrm{~A}, \mathrm{~B}, \mathrm{D}$.

\section{Anatomical structures, nomenclature, and abbreviations}

Anatomical structures were identified on the basis of cytoand myeloarchitecture and by their relative locations. Additional brain series stained for chemo- and immunoarchitecture (calcium-binding proteins parvalbumin, calbindin and calretinin, and acetylcholine-esterase, acetylcholine-transferase, NADPH-diaphorase and zinc in various combinations) were consulted to support the structural identification. High-resolution images of one of these series with neighboring sections stained for NADPH-diaphorase, AChE and cells (Nissl) are presented as supplementary material (https://doi. org $/ 10.12751 / \mathrm{g}$-node.8bbcxy). Examples for zinc stained sections are depicted in Hoffmann et al. (2008b).
Fig. 5 a First subplate of atlas plate 19. It consists of a montage of a Nissl-stained half-section with the mirrored adjacent myelin-stained half-section. The inset in the upper left corner indicates the anteriorposterior plate location in a lateral brain view which is also shown in numerical form relative to the most rostral "Start of Neocortex" as well as relative to the "Occipital crest" (in the lower right corner). b Second subplate of atlas plate 19. It combines the Nissl stained half section (left side) with delineations of the anatomical structures on the mirrored translucent (30\%) Nissl section (right side). Inset and coordinate indications as in (a). c Third subplate of atlas plate 19. It consists of an abbreviation list and the CT slice with overlaid contours of the anatomical structures. d Fourth subpanel of atlas plate 19. It shows the myelin-stained half section (left side) with Nissl-derived delineations of the anatomical structures superimposed onto the mirrored translucent (30\%) myelin-stained half section (right side). Inset and coordinate indications as in (a)

In general, since no unified neuroanatomical nomenclature exists to date (Swanson 2015), we have used the widely accepted Paxinos nomenclature and abbreviations for naming structures (as far as applicable) to ease comparison between species, including the rat (Paxinos and Watson 2007; Paxinos et al. 2009 and Zilles 1985 for cortex), the mouse (Franklin and Paxinos 2008; Watson and Paxinos 2010), the monkey (Paxinos et al. 2008), the gerbil (RadtkeSchuller et al. 2016) and the ferret (Radtke-Schuller et al. 2018). The already established terms for the AC fields of Phyllostomus discolor (Hoffmann et al. 2008b) were adopted and also mapped onto the atlas sections (borders marked by stars and field names are abbreviated in italic characters) and onto the brain surface (Fig. 6). Furthermore, we also adopted previous terms for auditory midbrain and brainstem nuclei for which bat specific terminology was already established. Abbreviations for nuclei and cortical regions are shown in uppercase characters, abbreviations for fiber tracts and fissures in lower case characters.

Delineations of functional and structural areas of the Phyllostomus discolor cortex in top and side views are depicted in Fig. 6. Outlines are horizontal and lateral projections of the atlas brain delineations in the plates.

An electronic version of the atlas plates, an index of abbreviations, an index of structures and the supplementary material is available at https://doi.org/10.12751 /g-node.8bbcxy.

Additional publications on bat neuroanatomy (and those implicitly including neuroanatomical data on bats) taken into account for comparison are listed in the separate bibliography below 'References'. 
(a)

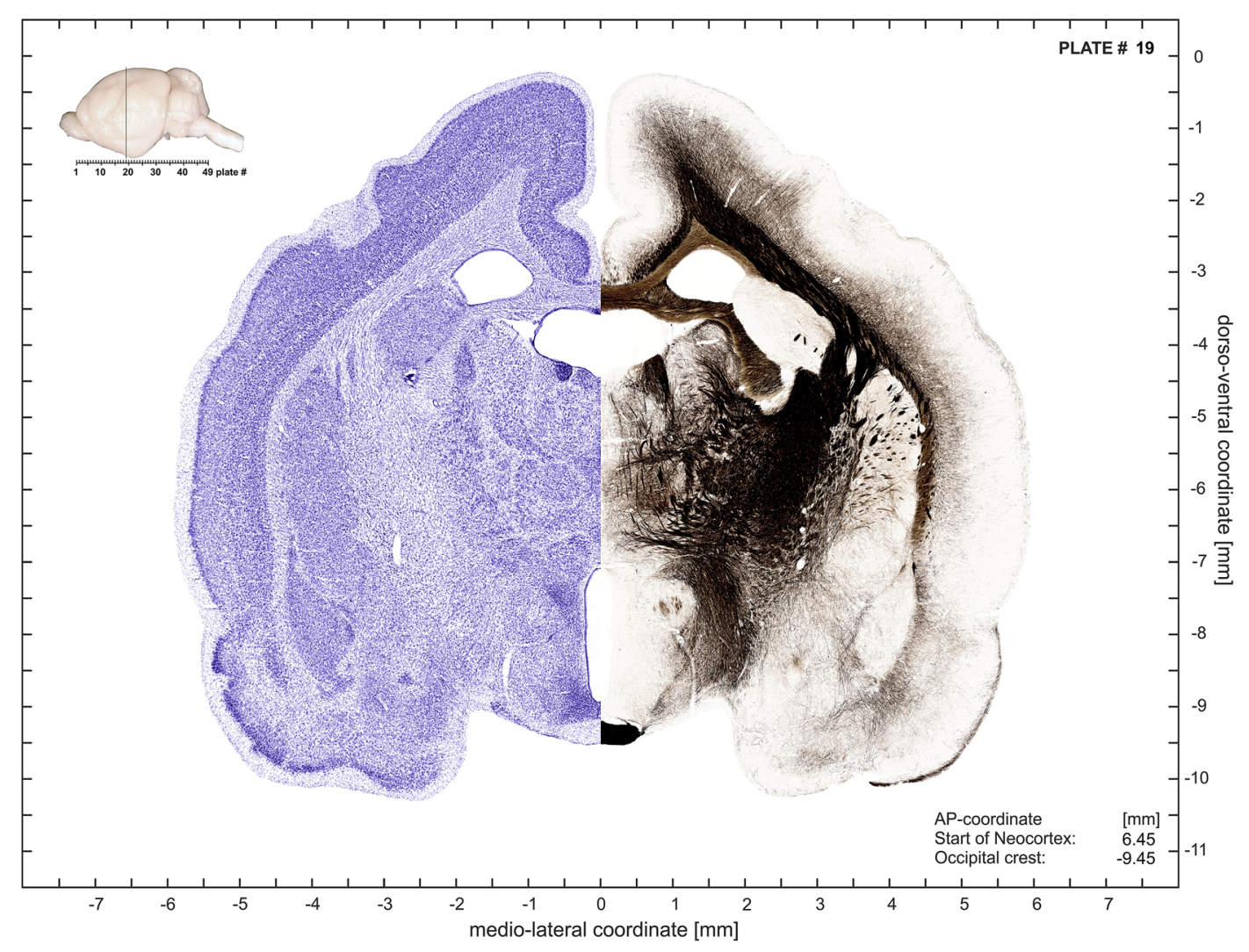

(b)

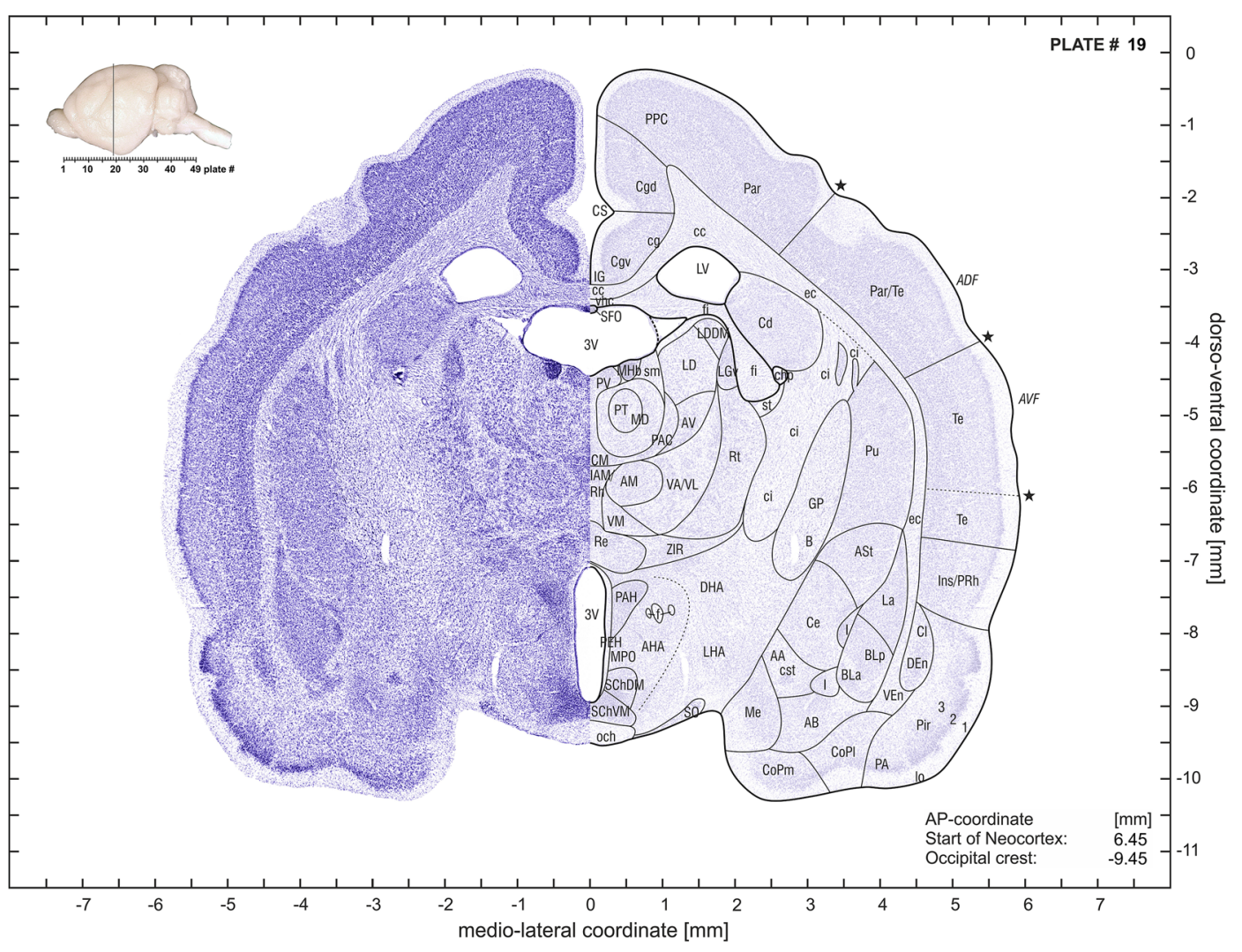


(c)

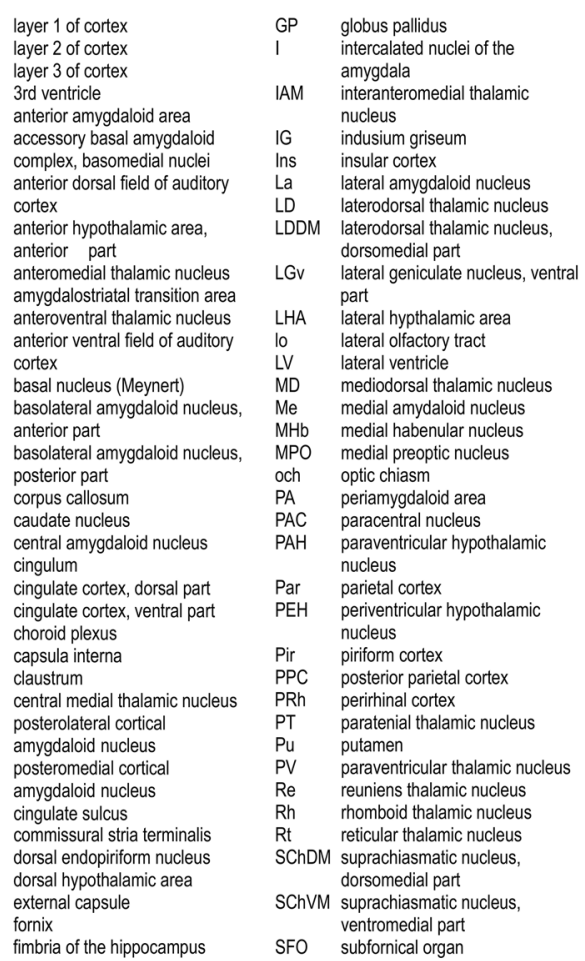

GP globus pallidus

nuclei of the

amygdala

IAM interanteromedial thalamic nucleus

IG indusium griseum

Ins insular cortex

La lateral amygdaloid nucleus

laterodorsal thalamic nucleus

laterodorsal thalamic nucleus,

LGv lateral geniculate nucleus, ventral

part

LHA lateral hypthalamic area

lo lateral olfactory tract

LV lateral ventricle

MD mediodorsal thalamic nucleus

medial amydaloid nucleus

$\mathrm{MHb}$ medial habenular nucleus

MPO medial preoptic nucleus

och optic chiasm

PA periamygdaloid area

PAC paracentral nucleus

PAH paraventricular hypothalamic

nucleus

Par parietal cortex

PEH periventricular hypothalamic

periventricul

Pir piriform cortex

PPC posterior parietal cortex

PRh perirhinal cortex

PT paratenial thalamic nucleus

$\mathrm{Pu}$ putamen

PV paraventricular thalamic nucleus

$\mathrm{Re}$ reuniens thalamic nucleus

$\mathrm{Rh}$ rhomboid thalamic nucleus

Rt reticular thalamic nucleus

SChDM suprachiasmatic nucleus,

dorsomedial part

SChVM suprachiasmatic nucleus,

ventromedial part

SFO subfornical organ

(d)

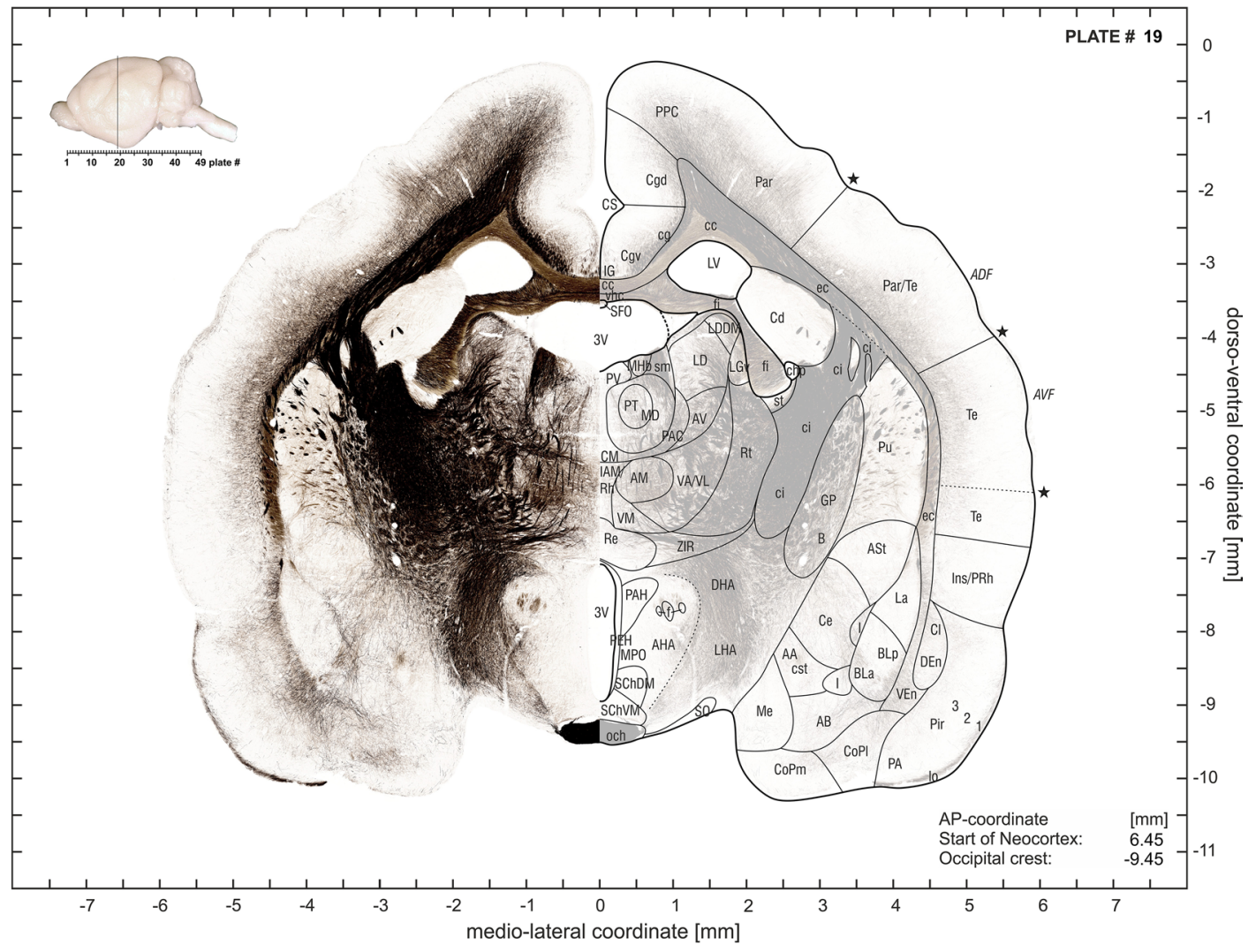

Fig. 5 (continued) 


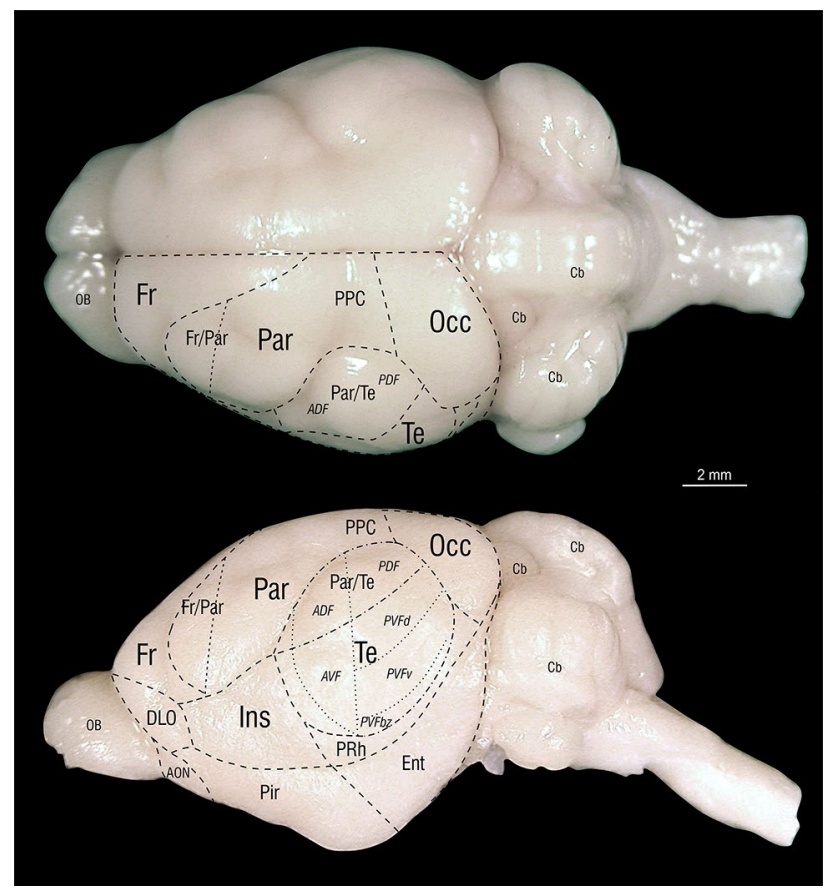

Fig. 6 Delineations of structural and functional areas of the Phyllostomus discolor cortex in top and side views. Outlines are horizontal and lateral projections of the atlas brain delineations. Functionrelated terms are in italic letters; anatomical cortical structures are in standard letters. Abbreviations: $A D F$ anterior dorsal auditory cortex field, AON anterior olfactory nuleus, $A V F$ anterior ventral auditory cortex field, $\mathrm{Cb}$ cerebellum, DLO dorsolateral orbital cortex, Ent entorhinal cortex, Fr frontal cortex, Fr/Par frontal-parietal transition zone, Ins insular cortex, OB olfactory bulb, Occ occipital cortex, Par parietal cortex, Par/Te parietal-temporal transition zone, $P D F$ posterior dorsal auditory cortex field, Pir piriform cortex, PPC posterior parietal cortex, PRh perirhinal cortex, $P V F b z$ border zone of posterior ventral auditory cortex field, $P V F d$ dorsal part of posterior ventral auditory cortex field, PVFv ventral part of posterior ventral auditory cortex field, Te temporal cortex

Acknowledgements The authors are grateful for the support and encouragement of late Karl Zilles, whose impressive anatomical work inspired the atlas work, Thomas Wachtler INCF G-Node (Germany) and Division of Neurobiology (Department of Biology II, LMU, Germany) for manpower, discussion and web space, Jonathan B. Fritz for correcting the English version of the manuscript, Matthias Starck for the possibility to use the virtual slide microscope in the Department of Biology II, LMU (Germany), the Anatomical Institute of the LudwigMaximilians-University Munich for lab space and Angelika Antonius, Anja Friedrich and Antonia Nekic for technical assistance. The authors gratefully acknowledge the funding sources Deutsche Forschungsgemeinschaft (DFG) ref. Schu390/7-1, Volkswagen Foundation (Auditory Object Normalization), and Human Frontier Science Program (HFSP) Research grant (RGP0058/2016).

Author contributions SRS: conceptualized atlas project, processed brains, imaged sections, matched sections with CT, labeled structures, created stereotaxic reference system, wrote, reviewed and edited manuscript. TF: supported atlas concept, perfused animals, reviewed and edited manuscript. HP: provided CT images of skull. GS: contributed to atlas concept, matched CT images, created stereotaxic reference system, prepared plates, wrote, reviewed and edited manuscript. UF: supported atlas concept, wrote, reviewed and edited manuscript.

Funding Open Access funding provided by Projekt DEAL.

\section{Compliance with ethical standards}

Conflict of interest The authors declare no competing financial interests.

Open Access This article is licensed under a Creative Commons Attribution 4.0 International License, which permits use, sharing, adaptation, distribution and reproduction in any medium or format, as long as you give appropriate credit to the original author(s) and the source, provide a link to the Creative Commons licence, and indicate if changes were made. The images or other third party material in this article are included in the article's Creative Commons licence, unless indicated otherwise in a credit line to the material. If material is not included in the article's Creative Commons licence and your intended use is not permitted by statutory regulation or exceeds the permitted use, you will need to obtain permission directly from the copyright holder. To view a copy of this licence, visit http://creativecommons.org/licenses/by/4.0/.

\section{References}

Baron G, Stephan H, Frahm HD (1996) Comparative Neurobiology in Chiroptera, vol 1. Basel Switzerland, Birkhäuser

Bartenstein SK, Gerstenberg N, Vanderelst D et al (2014) Echo-acoustic flow dynamically modifies the cortical map of target range in bats. Nat Commun 5:4668. https://doi.org/10.1038/ncommms566 8

Bhatnagar KP (2008) The brain of the common vampire bat, Desmodus rotundus murinus (Wagner 1840): a cytoarchitectural atlas. Braz J Biol 68:583-99. https://doi.org/10.1590/s1519-6984200800 0300017

Borina F, Firzlaff U, Schuller G, Wiegrebe L (2008) Representation of echo roughness and its relationship to amplitude-modulation processing in the bat auditory midbrain. Eur J Neurosci 27(10):2724 2732. https://doi.org/10.1111/j.1460-9568.2008.06247.x

Borina F, Firzlaff U, Wiegrebe L (2011) Neural coding of echo-envelope disparities in echolocating bats. J Comp Physiol A Neuroethol Sens Neural Behav Physiol 197(5):561-569. https://doi. org/10.1007/s00359-010-0571-z

Cyto- and myeloarchitectural brain atlas of the Ferret (Mustela putorius) in MRI Aided Stereotaxic Coordinates. Springer International Publishing, Cham. 10.1007/978-3-319-76626-3

Danscher G (1981) Histochemical demonstration of heavy metals. Histochemistry $71: 1-16$

Esser KH, Kiefer R (1996) Detection of frequency modulation in the FM-bat Phyllostomus discolor. J Comp Physiol A 178:787-96. https://doi.org/10.1007/bf00225827

Esser KH, Schmidt U (1989) Mother-infant communication in the lesser spear-nosed bat Phyllostomus discolor (Chiroptera, Phyllostomidae) - evidence for acoustic learning. Ethology 82:156-168

Esser KH (1994) Audio-vocal learning in a non-human mammal: the lesser spear-nosed bat Phyllostomus discolor. Neuroreport 5:1718-20. https://doi.org/10.1097/00001756-199409080-00007

Fenzl T, Schuller G (2002) Periaqueductal gray and the region of the paralemniscal area have different functions in the control of vocalization in the neotropical bat, Phyllostomus discolor. Eur J Neurosci 16:1974-86. https://doi.org/10.1046/j.1460-9568.2002.02261 
Fenzl T, Schuller G (2005) Echolocation calls and communication calls are controlled differentially in the brainstem of the bat Phyllostomus discolor. BMC Biol 3:17. https://doi. org/10.1186/1741-7007-3-17

Firzlaff U, Schuller G (2003) Spectral directionality of the external ear of the lesser spear-nosed bat, Phyllostomus discolor. Hear Res 185:110-122. https://doi.org/10.1016/S0378-5955(03)00164-3

Firzlaff U, Schörnich S, Hoffmann S, Schuller G, Wiegrebe L (2006) A neural correlate of stochastic echo imaging. J Neurosci. 26(3):78591. https://doi.org/10.1523/JNEUROSCI.3478-05.2006

Firzlaff U, Schuller G (2007) Cortical responses to object sizedependent spectral interference patterns in echolocating bats. Eur J Neurosci 26(10):2747-2755. https://doi.org/10.111 1/j.1460-9568.2007.05930.x

Firzlaff U, Schuchmann M, Grunwald JE et al (2007) Object-oriented echo perception and cortical representation in echolocating bats. PloS Biol 5(5):e100. https://doi.org/10.1371/journal.pbio.00501 00

Franklin KBJ, Paxinos G (2008) The mouse brain in stereotaxic coordinates, 3rd edn. Academic Press, London

Gallyas F (1979) Silver staining of myelin by means of physical development. Neurol Res 1:203-209. https://doi.org/10.1080/01616 412.1979.11739553

Genzel D, Hoffmann S, Prosch S, Firzlaff U, Wiegrebe L (2015) Biosonar navigation above water II: exploiting mirror images. J Neurophysiol 113(4):1146-1155. https://doi.org/10.1152/jn.00264.2014

Goerlitz HR, Hübner M, Wiegrebe L (2008) Comparing passive and active hearing: spectral analysis of transient sounds in bats. J Exp Biol 211:1850-8. https://doi.org/10.1242/jeb.017715

Greiter W, Firzlaff U (2017a) Echo-acoustic flow shapes object representation in spatially complex acoustic scenes. J Neurophysiol 117(6):2113-2124. https://doi.org/10.1152/jn.00860.2016

Greiter W, Firzlaff U (2017) Representation of three-dimensional space in the auditory cortex of the echolocating bat P. discolor. PLoS ONE 12(8):e0182461. https://doi.org/10.1371/journal.pone.01824 61

Hedreen JC, Bacon SJ, Price DL (1985) A modified histochemical technique to visualize acetylcholinesterase-containing axons. J Histochem Cytochem 33:134-140

Heinrich M, Warmbold A, Hoffmann S et al (2011) The sonar aperture and its neural representation in bats. J Neurosci 31(43):15618 15627. https://doi.org/10.1523/JNEUROSCI.2600-11.2011

Hörpel SG, Firzlaff U (2019) Processing of fast amplitude modulations in bat auditory cortex matches communication call-specific sound features. J Neurophysiol 121(4):1501-1512. https://doi. org/10.1152/jn.00748.2018

Hoffmann S, Baier L, Borina F, Schuller G, Wiegrebe L, Firzlaff U (2008) Psychophysical and neurophysiological hearing thresholds in the bat Phyllostomus discolor. J Comp Physiol A Neuroethol Sens Neural Behav Physiol 194(1):39-47. https://doi.org/10.1007/ s00359-007-0288-9

Hoffmann S, Firzlaff U, Radtke-Schuller S et al (2008) The auditory cortex of the bat Phyllostomus discolor: Localization and organization of basic response properties. BMC Neurosci 9:65. https:// doi.org/10.1186/1471-2202-9-65

Hoffmann S, Schuller G (2010) Dynamic stimulation evokes spatially focused receptive fields in bat auditory cortex. Eur J Neurosci 31(2):371-385. https://doi.org/10.1111/j.1460-9568.2009.07051.x

Hoffmann S, Warmbold A, Wiegrebe L, Firzlaff U (2013) Spatiotemporal contrast enhancement and feature extraction in the bat auditory midbrain and cortex. J Neurophysiol 110(6):1257-1268. https:// doi.org/10.1152/jn.00226.2013

Hoffmann S, Genzel D, Prosch S, Baier L, Weser S, Wiegrebe L (2015) Biosonar navigation above water I: estimating flight height. J Neurophysiol 113(4):1135-1145. https://doi.org/10.1152/jn.00263 .2014
Hoffmann S, Vega-Zuniga T, Greiter W, Krabichler Q, Bley A, Matthes M, Zimmer Ch, Firzlaff U, Luksch H (2016) Congruent representation of visual and acoustic space in the superior colliculus of the echolocating bat Phyllostomus discolor. Eur J Neurosci 44:2685-2697. https://doi.org/10.1111/ejn.13394

Hoffmann S, Bley A, Matthes M et al (2019) The neural basis of dimlight vision in echolocating bats. Brain Behav Evol 94:61-70. https://doi.org/10.1159/000504124

Janik VM, Slater PJB (1997) Vocal learning in mammals. Adv Study Behav 26:59-99. Academic Press, London ISBN 0-12-004526-5

Knörnschild M (2014) Vocal production learning in bats. Curr Opin Neurobiol 28:80-85. https://doi.org/10.1016/j.conb.2014.06.014

Kugler K, Luksch H, Peremans H et al (2019) Optic and echo-acoustic flow interact in bats. J Exp Biol 222(Pt 6):jeb195404. https://doi. org/10.1242/jeb. 195404

Kwiecinski GG (2006) Mammalian Species. Phyllostomus discolor 801:1-11. https://doi.org/10.1644/801.1

Lattenkamp EZ, Vernes SC, Wiegrebe L (2018) Volitional control of social vocalisations and vocal usage learning in bats. J Exp Biol. https://doi.org/10.1242/jeb.180729

Linnenschmidt M, Wiegrebe L (2019) Ontogeny of auditory brainstem responses in the bat, Phyllostomus discolor. Hear Res 373:85-95. https://doi.org/10.1016/j.heares.2018.12.010

Paxinos G, Watson C (2007) The rat brain in stereotaxic coordinates, 6th edn. Academic Press, San Diego (978-0-12-547612-4)

Paxinos G, Watson C, Carrive P et al (2009) Chemoarchitectonic atlas of the rat brain, 2nd edn. Academic Press, London (0123742377)

Paxinos G, Huang X-F, Petrides M, Toga AW (2008) The rhesus monkey brain, 2nd edn. Elsevier LTD, Oxford (978-0-12-373619-2)

Pirlot P, Stephan H (1970) Encephalization in Chiroptera. Can J Zool 48:433-444. https://doi.org/10.1139/z70-075

Radtke-Schuller S, Schuller G, Angenstein F et al (2016) Brain atlas of the Mongolian gerbil (Meriones unguiculatus) in CT/MRIaided stereotaxic coordinates. Brain Struct Funct. https://doi. org/10.1007/s00429-016-1259-0

Rodenas-Cuadrado PM, Chen XS, Wiegrebe L (2015) A novel approach identifies the first transcriptome networks in bats: a new model for vocal communication. BMC Genom. https://doi. org/10.1186/s12864-015-2068-1

Rodenas-Cuadrado PM, Mengede J, Baas L et al (2018) Mapping the distribution of language related genes FoxP1, FoxP2 and CntnaP2 in the brains of vocal learning bat species. J Comp Neurol 526:1235-1266. https://doi.org/10.1002/cne.24385

Rother G, Schmidt U (1982) The influence of visual information on echolocation in Phyllostomus-Discolor (Chiroptera). Z Saugetierkd 47:324-334

Scalia F, Rasweiler JJ, Scalia J et al (2013) Forebrain atlas of the shorttailed fruit bat Carollia perspicillate. Springer, Heidelbergy

Swanson LW (2015) Brain maps online: Toward open access atlases and a pan-mammalian nomenclature. J Comp Neurol 523:22722276. https://doi.org/10.1002/cne.23788

Schneider R (1957) Morphologische Untersuchungen am Gehirn der Chiropteren (Mammalia). Abh Senckenb Naturforsch Ges 495:1-92

Schneider R (1966) Das Gehirn von Rousettus aegyptiacus (E. Geoffroy 1810) (Megachiroptera, Chiroptera, Mammalia). Ein mit Hilfe mehrerer Schnittserien erstellter Atlas. Abh Senckenb Naturforsch Ges 513:1-160

Schuller G, Radtke-Schuller S, Betz M (1986) A stereotaxic method for small animals using experimentally determined reference profiles

Stephan H, Pirlot P (1970) Volumetric comparisons of brain structures in bats. J Zoolog System Evolution Res 8(1):200-236

Tyack PL (2020) A taxonomy for vocal learning. Philosophical Transactions of the Royal Society B Biological Sciences 375(1789):20180406. https://doi.org/10.3389/fnins.2020.00322 /rstb.2018.0406 
Vanderelst D, De Mey F, Peremans H et al (2010) What noseleaves do for FM bats depends on their degree of sensorial specialization. PLoS One 5:e11893. https://doi.org/10.1371/journ al.pone.0011893

Vernes SC, Wilkinson GS (2020) Behaviour, biology and evolution of vocal learning in bats. Philos Trans R Soc Lond B Biol Sci 375(1789):20190061. https://doi.org/10.1098/rstb.2019.0061

Vincent SR, Kimura H (1992) Histochemical mapping of nitricoxide synthase in the rat brain. Neuroscience 46:755-785

Wagner A (1843) Diagnosen neuer Arten brasilischer Handflügler. Archiv für Naturgeschichte 1:365-368

Washington SD, Hamaide J, Jeurissen B et al (2018) A threedimensional digital neurological atlas of the mustached bat (Pteronotus parnellii). Neuroimage 183:300-313. https://doi. org/10.1016/j.neuroimage.2018.08.013

Watson C, Paxinos G (2010) Chemoarchitectonic atlas of the mouse brain. Academic Press, London

Zilles K (1985) The cortex of the rat. Springer, Heidelberg 3642705758

\section{Additional publications on bat neuroanatomy (and those implicitly including neuroanatomical data on bats)}

Aschoff A, Ostwald J (1987) Different origins of cochlear efferents in some bat species, rats, and guinea pigs. J Comp Neurol 264:5672. https://doi.org/10.1002/cne.902640106

Baker RJ, JK Jones Jr, DC Carter (1976) Biology of Bats of the New World Family Phyllostomatidae. Part I. Special publications - The Museum, Texas Tech University; no. 10:1-216

Behrend O, Schuller G The central acoustic tract and audio-vocal coupling in the horseshoe bat, Rhinolophus rouxi

Bhatnagar KP, Smith TD, Rai SN, Frahm HD (2016) The Chiropteran Brain Database: Volumetric Survey of the Hypophysis in 165 Species. Anat Rec 299:492-510. https://doi.org/10.1002/ar.23321

Casseday J, Kobler J, Isbey S, Covey AE (1989) Central acoustic tract in an echolocating bat: an extralemniscal auditory pathway to the thalamus. J Comp Neurol 287:247-259. https://doi.org/10.1002/ cne. 902870208

Chang H-T (1944) High level decussation of the pyramids in the pangolin (Manis pentadactyla Dalmanni). J Comp Neurol 81:333-338. https://doi.org/10.1002/cne.900810307

Cotter JR, Pierson Pentney RJ (1979) Retinofugal projections of nonecholocating (Pteropus giganteus) and echolocating (Myotis lucifugus) bats. J Comp Neurol 184:381-399. https://doi. org/10.1002/cne.901840211

Covey E, Hall WC, Kobler JB (1987) Subcortical connections of the superior colliculus in the mustache bat, Pteronotus parnellii. J Comp Neurol 263:179-197. https://doi.org/10.1002/cne.90263 0203

del Campo HM, Measor K, Razak KA (2014) Parvalbumin and calbindin expression in parallel thalamocortical pathways in a gleaning bat, Antrozous pallidus. J Comp Neurol 522:2431-2445. https ://doi.org/10.1002/cne.23541

Frahm HD, War K, Bhatnagar P (1980) Comparative morphology of the accessory olfactory bulb in bats. J Anat 130(2):349-365

Gatome CW, Slomianka L, Mwangi DK et al (2010) The entorhinal cortex of the Megachiroptera: A comparative study of Wahlberg's epauletted fruit bat and the straw-coloured fruit bat. Brain Struct Funct 214:375-393. https://doi.org/10.1007/s00429-010-0239-z

Gibbons I, Sundaram V, Adogwa A, Odekunle A (2019) Cytoarchitecture of the superior olivary complex of three neotropical species of bats (Noctilio leporinus Phyllostomus hastatus and Carollia perspicillata) with different foraging behavior. Brazilian J Biol. https://doi.org/10.1590/1519-6984.210489

Gordon M, O'Neill WE (2000) An extralemniscal component of the mustached bat inferior colliculus selective for direction and rate of linear frequency modulations. J Comp Neurol 426:165-181

Grothe B, Park TJ (2000) Structure and function of the bat superior olivary complex. Microsc Res Tech 51:382-402

Grothe B, Schweizer H, Pollak GD et al (1994) Anatomy and Projection Patterns of the Superior Olivary Complex in the Mexican Free-Tailed Bat, Tadarida brasiliensis mexicana. J Comp Neurol 343:630-646

Heffner R, Masterton B (1975) Brain, Behavior and Evolution variation in form of the pyramidal tract and its relationship to digital dexterity'. Brain Behav Evol 12:161-200

Humphrey T (1936) The telencephalon of the bat. I. The non-nuclear masses and certain pertinent fiber connections. J Comp Neurol 65:603-711

Ito T, Furuyama T, Hase K et al (2018a) Organization of projection from brainstem auditory nuclei to the inferior colliculus of Japanese house bat (Pipistrellus abramus). Brain Behav. https://doi. org/10.1002/brb3.1059

Ito T, Furuyama T, Hase K et al (2018b) Organization of subcortical auditory nuclei of Japanese house bat (Pipistrellus abramus) identified with cytoarchitecture and molecular expression. J Comp Neurol 526:2824-2844. https://doi.org/10.1002/cne.24529

Ito T, Yamamoto R, Furuyama T et al (2019) Three forebrain structures directly inform the auditory midbrain of echolocating bats. Neurosci Lett. https://doi.org/10.1016/j.neulet.2019.134481

Kobler JB, Isbey SF, Casseday JH (1987) Auditory pathways to the frontal cortex of the mustache bat, Pteronotus parnellii. Science 236:824-826. https://doi.org/10.1126/science.2437655

Ma J, Kanwal JS (2014) Stimulation of the basal and central amygdala in the mustached bat triggers echolocation and agonistic vocalizations within multimodal output. Front Physiol. https:// doi.org/10.3389/fphys.2014.00055

Marsh RA, Fuzessery ZM, Grose CD, Wenstrup JJ (2002) Projection to the Inferior Colliculus from the Basal Nucleus of the Amygdala. Neuroscience 22(23): 10449-10460

Medeiros HHA, Santana MAD, Leite MD et al (2016) The cytoarchitectonic and $\mathrm{TH}$-immunohistochemical characterization of the dopamine cell groups in the substantia nigra, ventral tegmental area and retrorubral field in a bat (Artibeus planirostris). Neurosci Res 112:37-46. https://doi.org/10.1016/j.neures.2016.06.005

Metzner W (1996) Anatomical basis for audio-vocal integration in echolocating horseshoe bats. J Comp Neurol 368:252-269

Metzner W, Radtke-Schuller S (1987) The nuclei of the lateral lemniscus in the rufous horseshoe bat, Rhinolophus rouxi - A neurophysiological approach. J Comp Physiol A 160:395-411. https:// doi.org/10.1007/BF00613029

Naumann RT, Kanwal JS (2011) Basolateral amygdala responds robustly to social calls: spiking characteristics of single unit activity. Ghazanfar Hauser 105:2389-2404. https://doi.org/10.1152/ jn.00580.2010

Orman R, Kollmar R, Stewart M (2017) Claustrum of the short-tailed fruit bat, Carollia perspicillata: Alignment of cellular orientation and functional connectivity. J Comp Neurol 525:1459-1474. https ://doi.org/10.1002/cne.24036

Padova DM (2019) Batlas: variational reconstruction of a digital, threedimensional atlas of the big brown bat (Eptesicus fuscus) (Doctoral dissertation, Johns Hopkins University)

Peterson DC, Wenstrup JJ (2012) Selectivity and persistent firing responses to social vocalizations in the basolateral amygdala. Neuroscience 217:154-171. https://doi.org/10.1016/j.neuroscien ce.2012.04.069

Prasada Rao PD, Kanwal JS (2004) Oxytocin and Vasopressin Immunoreactivity within the Forebrain and Limbic-Related Areas in the 
Mustached Bat, Pteronotus parnellii. Brain Behav Evol 63:151168. https://doi.org/10.1159/000076241

Radtke-Schuller S (2001) Neuroarchitecture of the auditory cortex in the rufous horseshoe bat (Rhinolophus rouxi ). Anat Embryol (Berl) 203:81-100. https://doi.org/10.1007/s004290100191

Radtke-Schuller S (2004) Cytoarchitecture of the medial geniculate body and thalamic projections to the auditory cortex in the rufous horseshoe bat (Rhinolophus rouxi): I. Temporal fields Anat Embryol (Berl) 209:59-76. https://doi.org/10.1007/s0042 9-004-0424-z

Radtke-Schuller S, Schuller G, O'Neill WE (2004) Thalamic projections to the auditory cortex in the rufous horseshoe bat (Rhinolophus rouxi): II. Dorsal fields Anat Embryol (Berl) 209:77-91. https://doi.org/10.1007/s00429-004-0425-y

Radtke-Schuller S, Fenzl T, Peremans H, Schuller G, Firzlaff U (2020) Plates of Cyto- and Myeloarchitectural Brain Atlas of the Pale Spear-Nosed Bat (Phyllostomus discolor) and high-resolution images of sections stained for NADPH-diaphorase, Acetylcholine-esterase and Nissl. G-Node. https://doi.org/10.12751 /g-node.8bbcxy

Reimer K (1989) Retinofugal projections in the rufous horseshoe bat, Rhinolophus rouxi. Anat Embryol (Berl) 180:89-98

Rübsamen R, Schweizer H (1986) Control of echolocation pulses by neurons of the nucleus ambiguus in the rufous horseshoe bat, Rhinolophus rouxi. II Afferent and efferent connections of the motor nucleus of the laryngeal nerves. J Comp Physiol A 159:689-699

Santana MAD, Medeiros HHA, Leite MD et al (2018) Retinofugal projections into visual brain structures in the bat Artibeus planirostris: A CTb Study. Front Neuroanat. https://doi.org/10.3389/ fnana.2018.00066

Santana NNM, Barros MAS, Medeiros HHA et al (2018) The suprachiasmatic nucleus and the intergeniculate leaflet of the flat-faced fruit-eating bat (Artibeus planirostris): Retinal projections and neurochemical anatomy. Front Neuroanat. https://doi.org/10.3389/ fnana.2018.00036

Scalia F, Rasweiler JJ, Danias J (2015) Retinal projections in the shorttailed fruit bat, Carollia perspicillata, as studied using the axonal transport of cholera toxin B subunit: Comparison with mouse. J Comp Neurol 523:1756-1791. https://doi.org/10.1002/cne.23723

Schuller G, Radtke-Schuller S (1990) Neural control of vocalization in bats: mapping of brainstem areas with electrical microstimulation eliciting species-specific echolocation calls in the rufous horseshoe bat. Exp Brain Res 79:192-206. https://doi.org/10.1007/ BF00228889

Schweizer H (1981) The connections of the inferior colliculus and the organization of the brainstem auditory system in the greater horseshoe bat (Rhinolophus ferrumequinum). J Comp Neurol 201:25-49. https://doi.org/10.1002/cne.902010104

Smith JB, Alloway KD, Hof PR et al (2019) The relationship between the claustrum and endopiriform nucleus: A perspective towards consensus on cross-species homology. J Comp Neurol 527:476-499

Vater M, Braun K (1994) Parvalbumin, calbindin D-28k, and calretinin immunoreactivity in the ascending auditory pathway of horseshoe bats. J Comp Neurol 341:534-558. https://doi.org/10.1002/ cne. 903410409

Vater M, Feng AS (1990) Functional organization of ascending and descending connections of the cochlear nucleus of horseshoe bats. J Comp Neurol 292:373-395. https://doi.org/10.1002/cne.90292 0305

Winer JA, Wenstrup JJ (1994) Cytoarchitecture of the medial geniculate body in the mustached bat (Pteronotus parnellii). J Comp Neurol 346:161-182. https://doi.org/10.1002/cne.903460202

Wohlgemuth MJ, Kothari NB, Moss CF (2018) Functional organization and dynamic activity in the superior colliculus of the echolocating bat, Eptesicus fuscus. J Neurosci 38:245-256. https://doi. org/10.1523/JNEUROSCI.1775-17.2017

Yamamoto S, Shimoda B, Momma R, Sai H (1955) Studies on brain stem. Comparative anatomical study of brain of some species of bats. Tohoku J Experim Med 61(4):339-344

Yamamoto S, Kutsuzawa Y, Takashima K, Saito M(1955) On intrapontine facial nerves of some species of bats. Anat Lab Prof H Seto, Tohoku Univ Sendai 411-421

Zettel ML, Carr CE, O’Neill WE (1991) Calbindin-like immunoreactivity in the central auditory system of the mustached bat, Pteronotus parnellii. J Comp Neurol 313:1-16. https://doi.org/10.1002/ cne.903130102

Zook JM, Casseday JH (1982) Cytoarchitecture of auditory system in lower brainstem of the mustache bat, Pteronotus parnellii. J Comp Neurol 207:1-13. https://doi.org/10.1002/cne.902070102

Zook JM, Winer JA, Pollak GD, Bodenhamer RD (1985) Topology of the central nucleus of the mustache bat's inferior colliculus: Correlation of single unit properties and neuronal architecture. J Comp Neurol 231:530-546. https://doi.org/10.1002/cne.90231 0410

Publisher's Note Springer Nature remains neutral with regard to jurisdictional claims in published maps and institutional affiliations. 